\title{
Caffeine: Public Concern and the Precautionary Principle
}

\author{
Jack E. James
}

$\mathbf{T}$ HIS ISSUE OF THE Journal of Caffeine Research marks the beginning of our third year of operation. It is an occasion to reflect with gratitude upon the support we have received from members of the Editorial Board; the Editorial Office of our publishers, Mary Ann Liebert, Inc.; authors of articles; the many reviewers who enable us to sustain a healthy peer-review process; and of course subscribers and readers, many of whom have been very generous in their expressions of appreciation for the Journal. Above all, it is an occasion to renew our collective effort to ensure the continued excellence of the Journal.

Excellence for any scholarly journal depends in part on success in bringing matters of public importance to the attention of audiences beyond the scientific community. In this regard, the Journal can lay claim to having excelled. Many articles published in the first two volumes have received wide coverage in the popular print and electronic media, and the Journal's contributors frequently participate in public-media discussions about caffeine-related concerns.

Of the many caffeine-related concerns picked up recently by the media, possibly none has been more intensely debated than the consumption of caffeine energy drinks by children and adolescents (see this Journal's Editorial in Volume $2(4)) .^{1}$ In addition to being widely discussed in the public media, concerns have also been raised by elected public representatives, and investigated by national human services agencies in many countries. An illustration of that can be found in recent correspondence between elected representatives of the United States Senate and the United States Food and Drug Administration (FDA). ${ }^{2-5}$ Expressing concern about potential safety issues associated with the consumption, especially by young people, of energy drinks, Senators have petitioned the FDA to expand its investigatory and regulatory actions concerning caffeine.

A feature of FDA responses to questions in the aforementioned correspondence is a seeming reluctance to acknowledge harm despite evidence that harm exists. Specifically, FDA responses to questions from Senators includes the claim that for "healthy adults, caffeine intake up to $400 \mathrm{mg}$ per day $(\mathrm{mg} / \mathrm{d})$ is not associated with...cardiovascular effects [or] changes in adult behavior [and in a selective reference, absence of harm is claimed for] effects on male fertility" (italics added). ${ }^{3}$ The first two of these assertions is false, and the third is potentially misleading. The assertion that caffeine is not associated with cardiovascular effects is false. Although the association between caffeine consumption and cardiovascular disease remains inconclusive, it has been shown conclusively that caffeine at dietary doses produces cardiovascular effects, most notably modest (yet potentially harmful) increases in blood pressure. ${ }^{6}$ Similarly, the claim that dietary caffeine has no effect on behavior is false. For example, it has been shown conclusively that dietary caffeine produces physical dependence, evidenced by physical and behavioral withdrawal effects. ${ }^{7}$ These occur following brief periods of caffeine abstinence, including overnight sleep.

The specific reference to male fertility in the FDA response is selective and potentially misleading because it omits mention of female fertility. The roundtable discussion in this issue of the Journal focuses on a recent large Norwegian study of dietary caffeine and pregnancy outcomes in almost 60,000 expectant mothers. ${ }^{8}$ The study, which is the most comprehensive and thorough of its kind undertaken to date, found that caffeine intake was robustly associated with lower birth weight. Though firmer in its findings than previous studies, the observed association between dietary caffeine and lower birth weight reported in the Norwegian study is neither surprising nor new. As early as 1997, a review of relevant literature concluded that there is "strong evidence that caffeine consumed during pregnancy contributes to lower birth weight" (p. 131). ${ }^{9}$

\section{The Precautionary Principle}

Compounding problems caused by FDA reluctance to acknowledge instances of confirmed caffeine-related harm, is its defensiveness when faced with scientific disagreement. While showing appropriate overall sensitivity to its regulatory and public-safety responsibilities, FDA responses to questions indicate willingness to accept doubt about harm as a ground for inaction, possibly reflecting the ambiguity often discernible in the opinions and actions of the general public in relation to caffeine. However, as a major regulatory authority, the FDA would be expected to show more deference to the precautionary principle that ordinarily prevails in circumstances of disagreement. The precautionary principle states that in the absence of scientific consensus regarding potential harm from a particular action, the burden of proof lies with those responsible for the action to demonstrate absence of harm. Accordingly, the precautionary principle requires purveyors of caffeine products to demonstrate absence of harm, especially in relation to new products marketed to vulnerable groups. In fact, because potential for caffeine-related harm is known to be persistent and widespread

Reykjavík University, Reykjavik, Iceland. 
(as in the examples of increased blood pressure, physical dependence, and low birth weight cited above), absence of harm obviously cannot be demonstrated. Thus, FDA regulatory inaction not only breaches the precautionary principle, it is entirely unfounded in fact.

As the eminent British epidemiologist, Geoffrey Rose, explained more than three decades ago, "a large number of people exposed to a low risk is likely to produce more cases than a small number of people exposed to a high risk" (p. 1849). ${ }^{10}$ Applied to dietary caffeine, Rose's maxim suggests there should be little or no population exposure to caffeine, especially amongst vulnerable populations, including expectant mothers, children, and adolescents. FDA reluctance to acknowledge real and substantiated instances of caffeine-related harm and inaction in the face of ambiguity about a plethora of additional potential instances of caffeine-related harm shows that the FDA's approach to caffeine has to date favored a course diametrically opposed to the precautionary principle that ordinarily applies in matters of public health and safety.

\section{References}

1. James JE. Coffee and mortality: urgent need for clinical trials to assess putative benefits and harms. J Caffeine Res 2012; 2:53-54.

2. Letter from Senator R. J. Durbin to M. Hamburg, Commissioner, FDA (April 3, 2012). Available at http://durbin.senate .gov/public/index.cfm/files/serve?File_id =6657cb3d-33a84108-8292-7139d0b3b712. Accessed February 3, 2013.

3. Letter from J. Ireland, Assistant Commissioner, FDA to Senator R. J. Durbin (August 10, 2012). Available at http:// durbin.senate.gov/public/index.cfm/files/serve?File_id = 17eadaa1-85e7-4ceb-a827-be244fbddfa5. Accessed February $3,2013$.
4. Letter from Senators R. J. Durbin and R. Blumethal to M. Hamburg, Commissioner, FDA (September 11, 2012). Available at http://durbin.senate.gov/public/index.cfm/files/serve?File_ id = fe44b78e-32ae-41a0-8a61-2ddf14ab95d1. Accessed February 3, 2013.

5. Letter from M. Mital, Acting Assistant Commissioner, FDA to Senator R. J. Durbin (November 21, 2012). http://durbin .senate.gov/public/index.cfm/files/serve?File_id $=60 \mathrm{fccdd} 9-$ 7e60-45d4-b529-4bf472cc6eee. Accessed February 3, 2013.

6. James JE. A critical review of dietary caffeine and blood pressure: relationship that should be taken more seriously. Psychosom Med 2004;66:63-71.

7. Juliano LM, Griffiths RR. A critical review of caffeine withdrawal: empirical validation of symptoms and signs, incidence, severity, and associated features. Psychopharmacology 2004;176:1-29.

8. James JE, Sengpiel V, Brantsaeter AL. Dietary caffeine and pregnancy outcomes: a roundtable discussion. J Caffeine Res 2013;3:3-8.

9. James JE. Coffee and mortality: urgent need for clinical trials to assess putative benefits and harms. J Caffeine Res 2012;2: 149-152.

10. Rose G. Strategy of prevention: lessons from cardiovascular disease. Br Med J 1981;282:1847-1851.

Address correspondence to: Jack E. James, PhD Reykjavík University Menntavegur 1 101 Reykjavík Iceland

E-mail: jack@ru.is 